\title{
Riesgos psicosociales en personal de asistencia de una clínica de tercer nivel de la ciudad de Cartagena de Indias en 2016
}

\author{
Risks in a third level care team clinic of Cartagena de Indias city in 2016
}

Edna Margarita Gómez Bustamanteㄹ, Ana Sofía Rodríguez Méndez², Kelly Johana Ordosgoitia Montero³, Martha Cecilia Rojas Lopez ${ }^{4}$, Carlos Alberto Severiche Sierra ${ }^{5}$

\section{Resumen}

Objetivo. determinar los riesgos psicosociales del personal asistencial de una clínica privada de tercer nivel de la ciudad de Cartagena de Indias. Método. se realizó un estudio descriptivo de corte transversal, la población objeto de estudio fue de cincuenta y siete trabajadores del área asistencial, se utilizó la batería de instrumentos para la evaluación de factores de riesgo psicosocial diseñada por el Ministerio de la Protección Social de Colombia. Resultados. el promedio de edad es de 34.2 años, $52 \%$ pertenece al nivel educativo técnico, con un predominio del género femenino. El estado civil predominante con un $46 \%$ es soltero, $44 \%$ viven en estrato 2. La mayoría (74\%) de los trabajadores considera que su jefe brinda siempre o casi la posibilidad de tener contacto con él. Siempre tienen buena relación y apoyo de sus compañeros, $61 \%$ considera que no tienen control sobre el tiempo y cantidad de trabajo que realizan. El pago del salario no es puntual y los trabajadores tienen muy pocas posibilidades de ascenso y además sienten que la empresa muy pocas veces se preocupa por su bienestar. La prevalencia de nivel más alto de riesgo correspondió a un $76 \%$ por exceso de carga laboral, por alta exigencia de esfuerzo mental un $75 \%$ y está relacionado con el poco control sobre el trabajo en un $57 \%$. Los trabajadores se exponen a jornadas laborales extensas, poco tiempo de descanso, cansancio y falta de sueńo por jornadas nocturnas, escasa interacción con la familia y el contacto constante con situaciones de extremo sufrimiento y dolor.

Palabras claves: Centros de salud, riesgos psicosociales, salud laboral.

\footnotetext{
1. Doctora en Ciencias de la Educación, Magíster en Enfermería, Especialista en Salud Familiar, Especialista en Educación con énfasis en

Evaluación, Enfermera. Universidad de Cartagena. Cartagena de Indias, Colombia.
2. Especialista en Salud Ocupacional, Enfermera. Universidad de Cartagena. Cartagena de Indias, Colombia.

3. Especialista en Salud Ocupacional, Administradora de Empresas. Universidad de Cartagena. Cartagena de Indias, Colombia.

4. Especialista en Salud Ocupacional, Enfermera. Universidad de Cartagena. Cartagena de Indias, Colombia.

5. Magíster en Desarrollo Sostenible y Medio Ambiente, Especialista en Ingeniería Sanitaria y Ambiental, Especialista en Seguridad y Salud en

el Trabajo, Químico, Doctorante en Ciencias. Universidad de Cartagena. Cartagena de Indias, Colombia.
} 


\begin{abstract}
Objective. to determine the psychosocial risks of care personnel in a private third-level clinic in the city of Cartagena de Indias. Method. a cross-sectional descriptive study was carried out, the study population consisted of fifty-seven workers in the health care area, a battery of instruments was used for the evaluation of psychosocial risk factors designed by the Ministry of Social Protection of Colombia. Results. the average age is 34.2 years, $52 \%$ belongs to the technical education level, with a predominance of the female gender. The predominant civil status with $46 \%$ is single, $44 \%$ live in stratum 2 . The majority (74\%) of the workers consider that their boss always provides or almost the possibility of having contact with him. Always have good relationship and support from their peers, $61 \%$ feel they have no control over the time and amount of work they do. The payment of the salary is not punctual and the workers have very little possibility of promotion and also feel that the company very rarely worries about their well-being. The prevalence of the highest level of risk corresponded to $76 \%$ due to an excess of workload, due to a high mental stress requirement of $75 \%$ and related to poor control over work by $57 \%$. Workers are exposed to long hours of work, little rest time, tiredness and lack of sleep for nightly hours, poor interaction with the family and constant contact with situations of extreme suffering and pain.
\end{abstract}

Keywords: Health institutions, psychosocial risks, occupational health.

\section{Introducción}

El trabajo es un elemento vital para las personas y para su desarrollo se requiere de adecuadas condiciones de salud. Existen factores de riesgo que afectan las condiciones del ambiente y de la organización del trabajo que pueden afectar al trabajador o generar un efecto negativo en la empresa (1).

Trabajar es una forma de ser del hombre y una manera de recibir remuneración, aunque pueda significar, también, una posibilidad de adolecer. Entre las dimensiones que caracterizan los locales de trabajo, aquellas relacionadas a la carga horaria pueden causar agravios a la salud de los trabajadores (2). La sobrecarga laborales provoca una gran variedad de efectos nocivos sobre la capacidad física y mental, incluyendo la ocurrencia de accidentes de trabajo y otros agravios (3).

La salud laboral presenta estadísticas de importante consideración a nivel mundial; aproximadamente 2.2 millones de muertes se encuentran relacionadas con el trabajo, 270 millones de personas se lesionan y 160 millones de personas enferman debido a causas laborales; así mismo a nivel económico, los costos debido a los accidentes de trabajo, enfermedades profesionales, indemnizaciones, días de trabajo perdidos, interrupciones en la producción, gastos médicos y otros, constituyen aproximadamente el $4 \%$ del Producto Interno Bruto mundial (4).

De acuerdo con Feldman et al. (5) una de las primeras definiciones sobre factores psicosociales laborales fueron realizadas por el Comité Mixto de la Organización Internacional del Trabajo (OIT) y la Organización Mundial de la Salud (OMS) en 1984, en la cual señalan que los factores psicosociales en el trabajo consisten en interacciones entre el trabajo, su medio ambiente, la satisfacción en el trabajo y las condiciones de su organización, por una parte, y por la otra, las capacidades del trabajador, sus necesidades, su cultura y su situación personal fuera del trabajo, todo lo cual, a través de percepciones y experiencias pueden influir en la salud y en el rendimiento y la satisfacción en el trabajo. 
La Organización Internacional del Trabajo (OIT) y la Organización Mundial de la Salud (OMS) los definen como "todas las interacciones entre el trabajo, su medio ambiente, la satisfacción en el empleo y las condiciones de su organización, por un lado, y por otro, las capacidades del empleado, sus necesidades, su cultura y su situación personal fuera del trabajo; todo lo cual, a través de percepciones y experiencias, influye en su salud y en su rendimiento", definición con mayor uso para describir a los factores de riesgo psicosociales laborales (6), (7), (8).

Los efectos perjudiciales para la salud de los trabajadores asociados a la exposición a factores de riesgo psicosocial han sido demostrados por diversas investigaciones. Éstas evidencian asociaciones importantes entre aspectos como el ambiente de trabajo, las relaciones laborales, la comunicación, los estilos de liderazgo, variables personales y ambientales, con patologías derivadas del estrés como el síndrome de Burnout, acoso laboral y alteraciones psicológicas de diferente naturaleza (9), (10), (11), (12).

Los trabajadores de salud, por las características de su trabajo que incluye situaciones desgastantes a las que se enfrentan en sus actividades cotidianas, con altas exigencias y múltiples factores psicosociales, así como la transferencia que reciben de sus pacientes, constituyen un grupo de alta vulnerabilidad para desarrollar problemas relacionados con el estrés (13).

Según Cruz et al. (3) la serie de agravios a la salud presentada por los trabajadores del área de la salud, durante el transcurrir de los años ha sido intensificada. Cambios generados por la globalización y por la apertura económica influenciaron a las personas, la sociedad y las organizaciones, que necesitaron transformar sus estructuras, actividades y procesos para seguir siendo competitivos. Sin embargo, modificaciones en la sociedad y en las relaciones de producción fueron acompañadas de transformaciones en las manifestaciones de sufrimiento y psicopatología de los individuos. En el área de la salud, trabajar durante muchas horas en ambientes laborales insalubres puede representar riesgos a la salud de los trabajadores. Entre las mujeres trabajadoras existe una interacción entre factores estresantes del trabajo y extra laborales, porque asocian las demandas de trabajo con las demandas sociales y familiares.

En el mismo sentido, en Colombia, la Primera Encuesta Nacional de Condiciones de Salud y Trabajo en el Sistema General de Riesgos Profesionales (14), encontró en mayor porcentaje factores de riesgo asociados a las condiciones ergonómicas y a los agentes psicosociales, entre los cuales se identificaron trabajo monótono y repetitivo, atención al público, acoso por parte de los superiores, compańeros o subordinados, cambio frecuente en el desarrollo de tareas y poca claridad en la definición de responsabilidades.

En un estudio realizado en Chile desarrollado en la capital y en instituciones de complejidad similar a la de nuestro caso, Ansoleaga y Castillo (15), encontraron en trabajadores de la salud que el $47 \%$ de los participantes perciben alta demanda psicológica, $46 \%$ bajo control de decisión, $61 \%$ escaso apoyo social; así mismo el $75 \%$ de los trabajadores reportaron desajuste entre el esfuerzo realizado y las recompensas recibidas.

Dada la importancia que ha cobrado en los últimos tiempos el estudio de los factores psicosociales laborales y con la finalidad de poder conocer las características y hacia dónde van orientadas las investigaciones en esta área, se planteó como principal objetivo de este estudio determinar los riesgos psicosociales en personal asistencial de la clínica de tercer nivel seleccionada.

\section{Metodología}

Se realizó un estudio descriptivo de corte transversal en una clínica privada de tercer nivel de complejidad de la ciudad de Cartagena de indias. 
La población fue integrada por 57 trabajadores asistenciales entre médicos, enfermeras y auxiliares de diferentes áreas de la clínica.

Dentro de los criterios de inclusión se tuvieron en cuenta a empleados del área asistencial con más de seis meses de labores en la empresa que desearon voluntariamente pertenecer a este trabajo.

Se utilizó la batería de instrumentos para la evaluación de factores de riesgo psicosocial diseñada por el Ministerio de la Protección Social de Colombia (16). De la cual se tomaron, la ficha de datos generales que consta de 18 preguntas, asociadas a los principales aspectos de las condiciones socio-demográficas y ocupacionales. De igual forma se utilizó el cuestionario de factores de riesgo psicosocial intralaboral (forma b) que consigna 97 preguntas, distribuidas en bloques que evalúan:

- 1 a 12: las condiciones ambientales del(los) sitio(s) o lugar(es) donde habitualmente realizan su trabajo.

- 13 a 15: cantidad de trabajo que tiene a cargo.

- 16 a 20: el esfuerzo mental que les exige su trabajo.

- 21 a 28: la jornada de trabajo.

- 9 a 37: las decisiones y el control que les permite su trabajo.

- 38 a 40: cualquier tipo de cambio que ocurra en su trabajo.

- 41 a 45: la información que la empresa les ha dado sobre su trabajo.

- 46 a 48: la formación y capacitación que la empresa les facilita para hacer su trabajo.

- 49 a 61: el o los jefes con quien tengan más contacto.

- 62 a 73: las relaciones con otras personas y el apoyo entre las personas de su trabajo.

- $\quad 74$ a 78: la información que reciben sobre su rendimiento en el trabajo.

- 79 a 88: la satisfacción, reconocimiento y la seguridad que le ofrece su trabajo.

- $\quad 89$ a 97: la atención a clientes y usuarios.
Para la evaluación del instrumento, los datos fueron procesados estadísticamente utilizando el Microsoft Excel 2013. En su análisis se empleó estadística descriptiva, principalmente la distribución de frecuencias absolutas y relativas, medidas de tendencia central y variabilidad (17).

En lo que respecta a los aspectos éticos se trabajó según lo establecido por la resolución No 008430 de 1993, por la cual se establecen las normas científicas, técnicas y administrativas para la investigación en salud.

\section{Resultados}

\section{Características socio-demográficas y laborales del personal asistencial perteneciente al estudio}

En la Tabla 1, se evidencia que en la población estudiada hay un $88 \%$ de sexo femenino y solo $12 \%$ pertenecen al sexo masculino, el $54 \%$ de los participantes tienen entre 26 a 35 ańos, $60 \%$ tiene entre 36 y 45 ańos, mientras que $7 \%$ tiene menos de 25 años. La mayoría de los participantes viven en zona urbana $89 \%$ y en menor proporción $11 \%$ en zona rural. El estado civil predominante es soltero $46 \%$, seguido de casado 35\%, y los que viven en unión libre $16 \%$. De los trabajadores 44\%vive en estrato $2,30 \%$ en estrato $3,18 \%$ en estrato 1 , mientras que la minoría $9 \%$ vive en estrato 4 . El nivel educativo predomínate es el técnico con 58\%. De los encuestados $16 \%$ tiene entre 1 y 5 ańos de antigüedad en la empresa, $18 \%$ tiene entre 6-10 años, 39\% tiene entre 11-15 ańos, mientras que $30 \%$ tiene más de 15 ańos de antigüedad. Trabaja en turnos de 10 horas $65 \%$, en turnos de 12 horas $23 \%$, y la minoría $12 \%$ trabaja en turnos de 9 horas. El total de los encuestados tiene tipo de contrato a término indefinido.

Tabla 1. Características socio-demográficas.

\begin{tabular}{|r|r|r|}
\hline Sexo & Frecuencia & Acumulado \\
\hline Masculino & 7 & $12 \%$ \\
\hline Femenino & 50 & $88 \%$ \\
\hline Total & 57 & $100 \%$ \\
\hline
\end{tabular}




\begin{tabular}{|c|c|c|}
\hline Sexo & Frecuencia & Acumulado \\
\hline$<25$ años & 4 & $7 \%$ \\
\hline 26-35 años & 41 & $72 \%$ \\
\hline 36-45 años & 7 & $12 \%$ \\
\hline$>45$ años & 5 & $9 \%$ \\
\hline Total & 57 & $100 \%$ \\
\hline Procedencia & Frecuencia & Acumulado \\
\hline Urbana & 51 & $89 \%$ \\
\hline Rural & 6 & $11 \%$ \\
\hline Total & 57 & $100 \%$ \\
\hline Procedencia & Frecuencia & Acumulado \\
\hline Casado & 20 & $35 \%$ \\
\hline Separado & 2 & $4 \%$ \\
\hline Soltero & 26 & $46 \%$ \\
\hline Unión libre & 9 & $16 \%$ \\
\hline Divorciado & 0 & $0 \%$ \\
\hline Viudo & 0 & $0 \%$ \\
\hline Total & 57 & $100 \%$ \\
\hline Estrato & Frecuencia & Acumulado \\
\hline Estrato 1 & 10 & $18 \%$ \\
\hline Estrato 2 & 25 & $44 \%$ \\
\hline Estrato 3 & 17 & $30 \%$ \\
\hline Estrato 4 & 5 & $9 \%$ \\
\hline Total & 50 & $100 \%$ \\
\hline Nivel Educativo & Frecuencia & Acumulado \\
\hline Profesional & 24 & $42 \%$ \\
\hline Técnico & 33 & $58 \%$ \\
\hline Total & 50 & $100 \%$ \\
\hline Antigüedad & Frecuencia & Acumulado \\
\hline 1-5 años & 8 & $14 \%$ \\
\hline 6-10 años & 10 & $18 \%$ \\
\hline 11-15 años & 22 & $39 \%$ \\
\hline$>15$ años & 17 & $30 \%$ \\
\hline Total & 50 & $100 \%$ \\
\hline Turnos & Frecuencia & Acumulado \\
\hline 9 horas & 7 & $12 \%$ \\
\hline 10 horas & 37 & $65 \%$ \\
\hline 12 horas & 13 & $23 \%$ \\
\hline Total & 50 & $100 \%$ \\
\hline
\end{tabular}

\begin{tabular}{|r|r|r|}
\hline $\begin{array}{r}\text { Tipo de } \\
\text { contrato }\end{array}$ & Frecuencia & Acumulado \\
\hline $\begin{array}{r}\text { A término } \\
\text { indefinido }\end{array}$ & 57 & $100 \%$ \\
\hline Temporal & 0 & $0 \%$ \\
\hline $\begin{array}{r}\text { Prestación de } \\
\text { servicio }\end{array}$ & 0 & $0 \%$ \\
\hline Total & 50 & $100 \%$ \\
\hline
\end{tabular}

Fuente: Investigadores.

\section{Exposición laboral a riesgo psicosocial}

En cuanto al dominio de demandas del trabajo en la dimensión demandas ambientales y de esfuerzo físico, tal como se evidencia en la Tabla 2, el $44,6 \%$ de los participantes considera que algunas veces están expuestos a factores de riesgos, el 43,6\% considera que siempre lo están y11.9\% considera que nunca están expuestos. En la dimensión demandas emocionales, $46.7 \%$ de los encuestados manifestó siempre estar expuestos a situaciones emocionales de tristeza, violencia o dolor, 46,6\% considera que solo algunas veces y respondió nunca estar expuestos el 6,19\%. En la dimensión de demandas cuantitativas, 76,6\% de la población manifestó siempre una alta exigencia, 22,5\% algunas veces $22,5 \%$ y nunca $2,8 \%$. En la dimensión demandas de la jornada de trabajo $77 \%$ respondió estar expuestos, no está expuesto $23 \%$. En la dimensión de influencia del trabajo sobre el entorno extralaboral, 64\% manifestó algunas veces estar expuestos a factores de riesgo, siempre estar expuestos $28 \%$ y nunca estar expuestos 8\%. En la dimensión de demandas de carga mental, $75 \%$ respondió que siempre se presenta una exigencia de esfuerzo mental para desarrollar su trabajo, 22\% respondió que algunas veces y nunca el 3\%. 
Tabla 2. Características relacionadas con las demandas ambientales y de esfuerzo, cantidad de trabajo a cargo, demanda de carga mental, demandas de la jornada de trabajo, influencia del trabajo sobre el entorno extra laboral y demandas emocionales.

\begin{tabular}{|c|c|c|c|c|c|c|}
\hline $\begin{array}{r}\text { Demandas ambientales y } \\
\text { de esfuerzo físico }\end{array}$ & Nunca & $\%$ & Algunas veces & $\%$ & Siempre & $\%$ \\
\hline PREG1 & 12 & 21,1 & 43 & 75,4 & 2 & 3,6 \\
\hline PREG2 & 2 & 3,5 & 47 & 82,4 & 8 & 14,1 \\
\hline PREG3 & 44 & 77,2 & 11 & 19,3 & 2 & 3,5 \\
\hline PREG4 & 0 & 0 & 5 & 8,8 & 52 & 91,2 \\
\hline PREG5 & 0 & 0 & 40 & 70,2 & 17 & 29,8 \\
\hline PREG6 & 1 & 1,8 & 49 & 85,9 & 7 & 12,3 \\
\hline PREG7 & 8 & 14 & 48 & 84,2 & 1 & 1,8 \\
\hline PREG8 & 12 & 21,1 & 41 & 71,9 & 4 & 7,1 \\
\hline PREG9 & 0 & 0 & 7 & 12,3 & 50 & 87,7 \\
\hline PREG10 & 2 & 3,5 & 6 & 10,5 & 49 & 85,9 \\
\hline PREG11 & 0 & 0 & 8 & 14 & 49 & 86 \\
\hline PREG12 & 0 & 0 & 0 & 0 & 57 & 100 \\
\hline TOTAL & 81 & $11,80 \%$ & 305 & $44,60 \%$ & 298 & $43,60 \%$ \\
\hline $\begin{array}{r}\text { Cantidad de trabajo a } \\
\text { cargo }\end{array}$ & Nunca & $\%$ & Algunas veces & $\%$ & Siempre & $\%$ \\
\hline PREG13 & 1 & 1,8 & 11 & 19,3 & 45 & 78,9 \\
\hline PREG14 & 0 & 0 & 18 & 31,6 & 39 & 68,4 \\
\hline PREG15 & 1 & 1,8 & 9 & 15,8 & 47 & 82,5 \\
\hline TOTAL & 2 & $1,20 \%$ & 38 & $22,20 \%$ & 131 & $76,60 \%$ \\
\hline $\begin{array}{r}\text { Demanda de carga } \\
\text { mental }\end{array}$ & Nunca & $\%$ & Algunas veces & $\%$ & Siempre & $\%$ \\
\hline PREG16 & 0 & 0 & 11 & 19,3 & 46 & 80,7 \\
\hline PREG17 & 0 & 0 & 4 & 7 & 53 & 93 \\
\hline PREG18 & 0 & 0 & 1 & 1,8 & 56 & 98,2 \\
\hline PREG19 & 8 & 14 & 44 & 77,2 & 5 & 8,8 \\
\hline PREG20 & 0 & 0 & 4 & 7 & 53 & 93 \\
\hline TOTAL & 8 & $2,80 \%$ & 64 & $22,40 \%$ & 213 & $74,70 \%$ \\
\hline $\begin{array}{r}\text { Demandas de la jornada } \\
\text { de trabajo }\end{array}$ & Nunca & $\%$ & Algunas veces & $\%$ & Siempre & $\%$ \\
\hline PREG21 & 8 & 14 & 30 & 52,6 & 19 & 33,4 \\
\hline PREG22 & 34 & 59,6 & 21 & 36,8 & 2 & 3,5 \\
\hline PREG23 & 0 & 0 & 11 & 19,3 & 46 & 80,7 \\
\hline PREG24 & 42 & 73,7 & 51 & 15,8 & 6 & 10,6 \\
\hline PREG33 & 28 & 49,1 & 25 & 43,9 & 4 & 7 \\
\hline PREG37 & 35 & 61,4 & 21 & 36,8 & 1 & 1,8 \\
\hline TOTAL & 147 & $43,00 \%$ & 159 & $34,20 \%$ & 78 & $22,80 \%$ \\
\hline $\begin{array}{r}\text { Influencia del trabajo } \\
\text { sobre el entorno extra } \\
\text { laboral }\end{array}$ & Nunca & $\%$ & Algunas veces & $\%$ & Siempre & $\%$ \\
\hline PREG25 & 2 & 3,5 & 30 & 52,6 & 25 & 43,9 \\
\hline
\end{tabular}




\begin{tabular}{|r|r|r|r|r|r|r|}
\hline PREG26 & 8 & 14 & 49 & 86 & 0 & 0 \\
\hline PREG27 & 4 & 7 & 53 & 92,9 & 0 & 0 \\
\hline PREG28 & 5 & 8,8 & 13 & 22,8 & 39 & 68,4 \\
\hline TOTAL & 19 & 8,3 & 145 & 63,6 & 64 & 28,1 \\
\hline Demandas emocionales & Nunca & $\%$ & Algunas veces & $\%$ & Siempre & $\%$ \\
\hline PREG99 & 6 & 10,5 & 46 & 80,7 & 5 & 8,8 \\
\hline PREG90 & 0 & 0 & 7 & 12,3 & 50 & 87,7 \\
\hline PREG91 & 0 & 0 & 11 & 19,3 & 46 & 80,7 \\
\hline PREG92 & 0 & 0 & 12 & 21,1 & 45 & 78,9 \\
\hline PREG93 & 1 & 1,8 & 6 & 10,5 & 50 & 87,7 \\
\hline PREG94 & 9 & 15,8 & 48 & 84,2 & 0 & 0 \\
\hline PREG95 & 8 & 14 & 48 & 84,2 & 1 & 1,8 \\
\hline PREG96 & 0 & 0 & 14 & 24,6 & 43 & 75,4 \\
\hline PREG97 & 10 & 17,5 & 47 & 82,5 & 0 & 0 \\
\hline TOTAL & 34 & 6,6 & 239 & 46,6 & 240 & 46,8 \\
\hline
\end{tabular}

Fuente: Investigadores.

En la Tabla 3, se muestran los aspectos relacionados con el dominio de liderazgo y relaciones sociales en el trabajo; en la dimensión características de liderazgo, el 74\% de los encuestados manifestó siempre tener contacto y apoyo de su jefe para la realización de su trabajo, y algunas veces el 26\%. En la dimensión de relaciones sociales en el trabajo, 74\% de los encuestados respondió siempre tener buenas relaciones y apoyo entre compańeros, el 18\% respondió algunas veces y nunca el 8\%. En la demanda de retroalimentación del desempeño, el 84\% manifestó siempre recibirla, 14\% respondió algunas veces y nunca el $2 \%$.

Tabla 3. Aspectos relacionados con contacto con el jefe, relaciones con otras personas, información sobre el rendimiento.

\begin{tabular}{|r|r|r|r|r|r|r|} 
Contacto con el jefe & Nunca & $\%$ & Algunas veces & $\%$ & Siempre & $\%$ \\
\hline PREG49 & 0 & 0 & 18 & 31,6 & 39 & 68,5 \\
\hline PREG50 & 0 & 0 & 12 & 21,1 & 45 & 79 \\
\hline PREG51 & 0 & 0 & 21 & 36,8 & 36 & 63,2 \\
\hline PREG52 & 0 & 0 & 31 & 54,4 & 26 & 45,7 \\
\hline PREG53 & 0 & 0 & 9 & 15,8 & 48 & 84,2 \\
\hline PREG54 & 0 & 0 & 18 & 31,6 & 39 & 68,4 \\
\hline PREG55 & 0 & 0 & 24 & 42,1 & 33 & 57,9 \\
\hline PREG56 & 0 & 0 & 23 & 40,4 & 34 & 59,7 \\
\hline PREG57 & 0 & 0 & 6 & 10,5 & 51 & 89,5 \\
\hline PREG58 & 0 & 0 & 1 & 1,8 & 56 & 98,3 \\
\hline PREG59 & 0 & 0 & 7 & 12,3 & 50 & 87,7 \\
\hline PREG60 & 0 & 0 & 15 & 26,3 & 42 & 73,7 \\
\hline PREG61 & 0 & 0 & 11 & 19,3 & 46 & 80,7 \\
\hline TOTAL & 0 & $0 \%$ & 196 & $26,50 \%$ & 545 & $73,60 \%$ \\
\hline
\end{tabular}




\begin{tabular}{|r|r|r|r|r|r|r|}
\hline $\begin{array}{r}\text { Relaciones con otras } \\
\text { personas }\end{array}$ & Nunca & $\%$ & Algunas veces & $\%$ & Siempre & $\%$ \\
\hline PREG62 & 0 & 0 & 3 & 5,3 & 54 & 94,8 \\
\hline PREG63 & 0 & 0 & 0 & 0 & 57 & 100 \\
\hline PREG64 & 0 & 0 & 6 & 10,5 & 51 & 89,4 \\
\hline PREG65 & 0 & 0 & 6 & 10,5 & 51 & 89,5 \\
\hline PREG66 & 51 & 89,5 & 5 & 8,8 & 1 & 1,8 \\
\hline PREG67 & 0 & 0 & 2 & 3,5 & 55 & 96,5 \\
\hline PREG68 & 0 & 0 & 33 & 57,9 & 24 & 42,2 \\
\hline PREG69 & 0 & 0 & 8 & 14,1 & 49 & 85,9 \\
\hline PREG70 & 0 & 0 & 10 & 17,5 & 47 & 82,4 \\
\hline PREG71 & 2 & 3,5 & 27 & 47,4 & 28 & 49,2 \\
\hline PREG72 & 0 & 0 & 8 & 14 & 49 & 85,9 \\
\hline PREG73 & 0 & 0 & 15 & 26,3 & 42 & 73,7 \\
\hline TOTAL & 53 & $7,80 \%$ & 123 & $18,00 \%$ & 508 & $74,30 \%$ \\
\hline Información sobre el & Nunca & $\%$ & Algunas veces & $\%$ & Siempre & $\%$ \\
\hline rendimiento & 1 & 1,8 & 15 & 26,3 & 41 & 71,9 \\
\hline PREG74 & 1 & 6 & 10,6 & 51 & 89,4 \\
\hline PREG75 & 0 & 0 & 2 & 3,6 & 54 & 94,8 \\
\hline PREG76 & 1 & 1,8 & 9 & 15,8 & 46 & 80,7 \\
\hline PREG77 & 2 & 3,5 & 8 & 14,1 & 47 & 82,4 \\
\hline PREG78 & 2 & 3,5 & 40 & 14,1 & 239 & 83,8 \\
\hline TOTAL & 6 & 2,1 & & & \\
\hline
\end{tabular}

Fuente: Investigadores.

Como se muestra en la Tabla 4, en el dominio de control sobre el trabajo, 90\% manifestó siempre tener claridad en el rol, 8\% algunas veces y $2 \%$ nunca. Recibe capacitación 62\%, algunas veces 36\% y nunca recibe $2 \%$. En la dimensión de participación y manejo del cambio, 58\% respondió tenerla, algunas veces $40 \%$ y nunca $2 \%$. En la dimensión de oportunidades para el uso y desarrollo de habilidades y conocimientos, 61\% respondió nunca tenerla, algunas veces 39\%. En la dimensión de control y autonomía sobre el trabajo, 57\% respondió nunca tenerlo, algunas veces 33\% y siempre 10\%

Tabla 4. Variables relacionadas con oportunidades de desarrollo y uso de habilidades y conocimientos, control y autonomía sobre el trabajo, participación y manejo del cambio, claridad de rol y capacitación.

\begin{tabular}{|r|r|r|r|r|r|r|}
\hline $\begin{array}{r}\text { Oportunidades de } \\
\text { desarrollo y uso } \\
\text { de habilidades y } \\
\text { conocimientos }\end{array}$ & Nunca & $\%$ & Algunas veces & $\%$ & Siempre & $\%$ \\
\hline PREG29 & 0 & 0 & 42 & 73,7 & 15 & 26,3 \\
\hline PREG30 & 0 & 0 & 20 & 35,1 & 37 & 64,9 \\
\hline PREG31 & 0 & 0 & 5 & 8,8 & 52 & 91,2 \\
\hline PREG32 & 0 & 0 & 22 & 38,6 & 35 & 61,5 \\
\hline TOTAL & 0 & $0 \%$ & 89 & $39,10 \%$ & 139 & $61,00 \%$ \\
\hline
\end{tabular}




\begin{tabular}{|r|r|r|r|r|r|r|}
\hline $\begin{array}{r}\text { Control y autonomía } \\
\text { sobre el trabajo }\end{array}$ & Nunca & $\%$ & Algunas veces & $\%$ & Siempre & $\%$ \\
\hline PREG34 & 35 & 61,4 & 22 & 38,6 & 0 & 0 \\
\hline PREG35 & 21 & 36,8 & 30 & 52,6 & 6 & 10,5 \\
\hline PREG36 & 41 & 71,9 & 4 & 7 & 12 & 21,1 \\
\hline TOTAL & 97 & 56,7 & 56 & 32,7 & 18 & 10,5 \\
\hline Participación y & Nunca & $\%$ & Algunas veces & $\%$ & Siempre & $\%$ \\
manejo del cambio & 0 & 0 & 15 & 26,3 & 42 & 73,7 \\
\hline PREG38 & 1 & 1,8 & 18 & 31,6 & 38 & 66,7 \\
\hline PREG39 & 2 & 3,5 & 35 & 61,4 & 20 & 35,1 \\
\hline PREG40 & 3 & $1,80 \%$ & 68 & $39,80 \%$ & 100 & $58,50 \%$ \\
\hline TOTAL & $\%$ & Algunas veces & $\%$ & Siempre & $\%$ \\
\hline Claridad de rol & Nunca & 2 & 3,5 & 55 & 96,5 \\
\hline PREG41 & 0 & 0 & 10 & 17,5 & 45 & 79 \\
\hline PREG42 & 2 & 3,5 & 0 & 0 & 53 & 93 \\
\hline PREG43 & 4 & 7 & 7 & 12,3 & 50 & 87,7 \\
\hline PREG44 & 0 & 0 & 3 & 5,3 & 54 & 94,7 \\
\hline PREG45 & 0 & 0 & 22 & $7,70 \%$ & 257 & $90,20 \%$ \\
\hline TOTAL & 6 & $2,10 \%$ & 6 & 10,6 & 51 & 89,5 \\
\hline Capacitación & Nunca & $\%$ & Algunas veces & $\%$ & Siempre & $\%$ \\
\hline PREG46 & 0 & 0 & 26 & 45,6 & 31 & 54,3 \\
\hline PREG47 & 0 & 0 & 30 & 52,6 & 23 & 40,4 \\
\hline PREG48 & 4 & 7 & 62 & $36,30 \%$ & 105 & $61,40 \%$ \\
\hline TOTAL & 4 & $2,30 \%$ & & &
\end{tabular}

Fuente: Investigadores.

La Tabla 5, señala que en el dominio de recompensas, 89\% respondió siempre a la dimensión recompensas derivadas de la pertenencia a la organización y del trabajo que se realiza, algunas veces $10 \%$ y nunca $1 \%$. No recibe reconocimiento y compensación $44 \%$, algunas veces $28 \%$ y siempre $28 \%$.

Tabla 5. Características de reconocimiento y compensación y recompensas derivadas de la pertenencia a la organización y del trabajo que se realiza.

\begin{tabular}{|r|r|r|r|r|r|r|}
$\begin{array}{r}\text { Reconocimiento y } \\
\text { compensación }\end{array}$ & Nunca & $\%$ & Algunas veces & $\%$ & Siempre & $\%$ \\
\hline PREG79 & 51 & 89,5 & 6 & 10,5 & 0 & 0 \\
\hline PREG80 & 1 & 1,8 & 1 & 1,8 & 55 & 96,5 \\
\hline PREG81 & 4 & 7 & 8 & 14,1 & 45 & 79 \\
\hline PREG82 & 19 & 33,3 & 30 & 52,7 & 8 & 14,1 \\
\hline PREG83 & 12 & 21,1 & 37 & 64,9 & 8 & 14 \\
\hline PREG84 & 16 & 28,1 & 32 & 56,2 & 9 & 15,8 \\
\hline TOTAL & 103 & 30,1 & 114 & 33,4 & 125 & 36,6 \\
\hline
\end{tabular}




\begin{tabular}{|r|r|r|r|r|r|r|}
\hline $\begin{array}{r}\text { Recompensas } \\
\text { derivadas de la } \\
\text { pertenencia a la }\end{array}$ & Nunca & $\%$ & Algunas veces & $\%$ & Siempre & $\%$ \\
$\begin{array}{r}\text { organización y del } \\
\text { trabajo que se realiza }\end{array}$ & & & & & & \\
\hline PREG85 & 0 & 0 & 1 & 1,8 & 56 & 98,3 \\
\hline PREG86 & 0 & 0 & 1 & 1,8 & 56 & 98,2 \\
\hline PREG87 & 1 & 1,8 & 18 & 31,6 & 38 & 66,7 \\
\hline PREG88 & 1 & 1,8 & 4 & 7 & 52 & 91,3 \\
\hline TOTAL & 2 & 0,9 & 24 & 10,6 & 202 & 88,6 \\
\hline
\end{tabular}

Fuente: Investigadores.

\section{Discusión}

Los resultados de esta investigación indican que en la población objeto de este estudio predomina el personal de sexo femenino. La edad sobresaliente se encuentra en el grupo de 26 a 35 años. Este estudio coincide con el realizado en la ciudad de Cartagena, titulado Estrés laboral en enfermería factores asociados (18), que utilizó la versión española del instrumento "The Nursing Stress Scale" (NSS) que arrojó resultados donde el grupo etario predomínate fue en promedio 33 años, donde casi el total de la población pertenecía al sexo femenino.

Así también se evidencia el estudio realizado por Hilleshein \& Lautert (19) acerca de la capacidad de trabajo, características socio-demográficas y laborales de las enfermeras de un hospital universitario en Río de Janeiro. El estudio transversal evaluó la capacidad de 195 enfermeras. Los datos fueron recogidos por el Índice de Instrumento Capacidad para el Trabajo y analizados por estadísticas y análisis y, 94,5\% de la muestra eran mujeres, edad media de 42,6 años. En nuestro caso los resultados son similares a este estudio lo cual podemos explicar por el tipo de ocupaciones a las que se dedica la mayoría de éstos, dado que hace parte de las ramas del sector salud donde tienen más afinidad las mujeres.

El resultado de esta investigación arroja que los trabajadores participantes se encuentran expuestos en un alto riesgo a diferentes factores relacionados con el dominio demandas del trabajo en las dimensiones de demandas cuantitativas, demandas de la jornada de trabajo, demandas de carga mental, donde más del $70 \%$ de los trabajadores consideran que siempre se encuentran expuestos a estos factores de riesgos. En la dimensión de influencia del trabajo sobre el entorno extra laboral, 64\% manifestó algunas veces estar expuestos a factores de riesgo y $28 \%$ siempre estar expuestos, considerándose un riesgo alto, ya que la mayoría de la población respondió que sí está expuesta. Esto evidencia que casi siempre el tiempo que tienen los empleados para realizar su actividades no es suficiente, y por tanto, deben extender su jornada laboral, las cuales son muy exigentes y extensas, quedando poco tiempo para sus familias y ámbito social, incluso en fines de semana y feriados.

Además, los resultados muestran que según las exigencias de sus labores deben trabajar en horarios nocturnos, y en días que normalmente deberían ser de descanso como los domingos y festivos. Lo anterior coincide con un estudio realizado por varios licenciados del Hospital Pediátrico Provincial "Paquito González Cueto" de Cienfuegos en Cuba (20) relacionado con los riesgos psicolaborales factores de riesgo Psicosocial en los que el mayor porcentaje fue agotamiento físico y mental relacionados con extensas jornadas de trabajo y poco descanso. Esto lo podemos explicar por el hecho de la naturaleza del ejercicio de las personas que laboran en el área asistencial de la salud, en los que la gran mayoría de las veces 
las demandas de éste le impiden a los trabajadores decidir cuánto tiempo pueden dedicar en una jornada laboral y las pocas posibilidades de pausas y momentos de descanso.

En el dominio de control sobre el trabajo se puede considerar que los participantes tienen claridad sobre sus roles y funciones, están capacitados y pueden aplicar sus conocimientos y habilidades. Sin embargo, en las dimensiones de oportunidades de desarrollo y de control y autonomía sobre el trabajo, se encuentra un alto riesgo ya que la mayoría de los participantes de este estudio manifestó tener poco control y autonomía sobre el trabajo y pocas posibilidades de progreso dentro de la empresa. Un estudio similar en Colombia realizado en la ciudad de Cali por Ortiz, F. A., \& Jaramillo, donde se investigaron los Factores De Riesgo Psicosocial y compromiso (Engagement) mostró relaciones estadísticamente significativas con los factores psicosociales de control sobre el trabajo (21). Una explicación a esto es que el hecho de las exigencias propias de los servicios en salud implican la necesidad de atender vidas humanas que se encuentran en vulnerabilidad y que no se puede en la mayoría de los casos planear las pausas, la cantidad y el orden de trabajo durante la jornada y mucho menos la velocidad a la que se pueda realizar, es necesario trabajar a ritmos muy acelerados en los que es imposible decidir por momentos de descanso, exigiéndole mucho esfuerzo y en algunos casos sobrecarga laboral.

En el dominio de recompensas, el estudio muestra un alto riesgo en la dimensión de reconocimiento y compensación, debido a que el pago del salario no es puntual y los trabajadores casi nunca tienen posibilidades de ascenso. Este resultado difiere con el realizado en Chile por el Ministerio de Salud de la Provincia de Córdoba (22) que arrojó riesgo medio en dimensión Compensaciones (39\%). Esto se puede explicar por el hecho de las pocas oportunidades de mejores empleos y salarios que ofrece el sector salud en Colombia, mientras que en Chile la situación salud es más desarrollada y con mejor remuneración y mayores oportunidades de progreso laboral.

En el dominio de liderazgo y relaciones sociales en el trabajo, se encuentra un bajo riesgo en todas sus dimensiones porque la mayoría de los participantes manifestó un adecuado ejercicio del liderazgo y tener buenas relaciones y apoyo con sus jefes y compañeros de trabajo. Así mismo la mayoría recibe retroalimentación sobre su rendimiento laboral. Este resultado es similar al arrojado por el estudio realizado por Castillo et al. (17) donde utilizaron el Cuestionario de evaluación de riesgos psicosociales del trabajo (ISTAS21), adaptación al español del Cuestionario psicosocial de Copenhague (CoPsoQ) a médicos de una Empresa Social del Estado (ESE) localizada en Cartagena de Indias, en el cual $88 \%$ presentó una exposición favorable para la salud en las dimensiones: apoyo social y calidad de liderazgo, lo cual resulta un factor positivo al momento de la resolución de problemáticas laborales y para la generación de un adecuado ambiente laboral que incida en la eficiencia, la productividad y la calidad de vida de los colaboradores de los diferentes equipos de trabajo.

Las limitaciones que tuvo esta investigación fue la poca colaboración de parte de los trabajadores para el desarrollo del instrumento (encuestas), para lo cual manifestaban que debido a su carga laboral no tenían espacio para dedicarle al desarrollo de éstas, lo cual dilató mucho el tiempo de aplicación del instrumento que se tenía planeado en la metodología. Esta exclusión desde el punto de vista cuantitativo restringe la realidad total de los riesgos psicosociales que afectan al personal de asistencia en la clínica de tercer nivel, a su vez desde el punto de vista cualitativo queda la incertidumbre en torno a la problemática psicolaboral en el personal de área de cirugía, lo que motiva a la realización de futuros estudios con esta población en particular.

Como conclusión, en el dominio y dimensiones de demandas del trabajo se evidencia que casi siempre el tiempo que tienen los empleados para 
realizar sus actividades no es suficiente, $y$ por tanto, deben extender sus jornadas laborales, las cuales son muy exigentes, quedando poco tiempo para sus familias y ámbito social, incluso en fines de semana y feriados. El esfuerzo mental realizado por los empleados es siempre o casi siempre muy exigente, lo que implica una mayor exigencia de concentración, memoria y responsabilidad.

En el dominio de liderazgo y relaciones sociales en el trabajo, el jefe brinda a sus trabajadores la posibilidad de tener contacto con él, de modo que estos tienen su apoyo y la ayuda necesaria que contribuya a desarrollar mejor su trabajo, así como solucionar los problemas que se presente en sus labores. De igual forma éste recibe información acerca de su rendimiento laboral, lo cual lo ayuda para estar en continuo mejoramiento del mismo. Existe una buena relación con las otras personas y apoyo mutuo para la realización del trabajo cuando se amerite. En el dominio control sobre el trabajo el empleado tiene claro su rol y considera que puede aprender y aplicar sus conocimientos en las decisiones que deba tomar en su trabajo diario, pero no tiene control sobre la cantidad y el tiempo que debe dedicar a este. La empresa brinda a sus trabajadores la oportunidad de capacitaciones que le permita realizar mejor su trabajo. (23-25).

En el dominio recompensas el pago del salario no es puntual y los trabajadores tienen muy pocas posibilidades de ascenso, sin embargo, su trabajo es estable y la mayoría se sienten orgullosos de trabajar en la empresa.

\section{Recomendaciones}

Se recomienda seguir profundizando en este tipo de estudios y tomando como población objeto todas las áreas de los servicios asistenciales de la institución, con el fin de obtener resultados más completos.
En futuras investigaciones, mayor apoyo de parte del área de talento humano para impulsar a sus trabajadores a brindar más colaboración al grupo investigador para el desarrollo del instrumento y así obtener mejores resultados para el beneficio de ella misma.

Desarrollar métodos de medición e investigación que brinden información que sirva de base para la identificación de los factores de riesgos y la toma de medidas de control y estrategias de prevención que disminuyan el estrés laboral.

\section{Referencias}

1. Bustillo M, Rojas J, Sanchez A, Sanchez L, Montalvo A, Rojas M. Riesgo psicosocial en el personal de enfermería. Servicio de urgencias en Hospital Universitario de Cartagena. Duazary. 2015; 12(1): 32-40.

2. Ceballos P, Valenzuela S, Paravic T. Factores de riesgos psicosociales en el trabajo: género y enfermería. av.enferm. 2014; 32(2): 271-279.

3. Cruz M, Chaves M, Barcellos R, Almeida L, de Oliveira A. Exceso de trabajo y agravios mentales a los trabajadores de la salud. Rev Cubana Enfermer. 2010; 26(1): 52-64.

4. Parra N, Pulido N. Riesgos psicosociales en una entidad pública de Boyacá. Revista de Psicología Universidad de Antioquia. 2013; 5(1): 59-73.

5. Feldman L, Blanco G. Una aproximación al estudio de los factores psicosociales laborales en Venezuela. Salud de los Trabajadores. 2012; 20(1): 75-92.

6. Aranda C, González R, Pando M, Hidalgo G. Factores de riesgo psicosocial laborales, apoyo social y síndrome de Burnout en médicos de familia de tres instituciones públicas de salud de Guadalajara (México). Salud Uninorte. 2013; 29(3): 487-500.

7. Canales M, Valenzuela S, Paravic T. Condiciones de trabajo de los profesionales de enfermería en Chile. Enfermería Universitaria. 2016; 13(3): 178-186.

8. Charria V, Sarsosa K, Arena F. Factores de riesgo psicosocial laboral: métodos e instrumentos de evaluación. Revista Facultad Nacional de Salud Pública. 2011; 29(4): 380-391.

9. García A, Gutiérrez M, Bellón J, Muñoz C, Fernández J. Entorno psicosocial y estrés en trabajadores sanitarios de la sanidad pública: diferencias entre atención primaria y hospitalaria. Atención Primaria. 2015; 47(6): 359-366.

10. Mcmillan K, Butow P, Turner J, Yates P, White K, Lambert S, Stephens M, Lawsin C. Burnout and the provision of psychosocial care amongst Australian cancer nurses. European Journal of Oncology Nursing. 2016; 22: 37-45. 
11. Galletta M, Portoghese I, D'Aloja E, Mereu A, Contu P, Coppola R, Finco G, Campagna M. Relationship between job burnout, psychosocial factors and health care-associated infections in critical care units. Intensive and Critical Care Nursing. 2016; 34: 59-66.

12. Talavera B, Luceño L, Martín J, Navarro A. Factores de riesgo psicosocial en médicos de la provincia de Valladolid: diferencias entre atención primaria y hospitalaria. Atención Primaria.2016; 48(6): 424-426.

13. Aldrete M, Navarro C, González R, Contreras M, Pérez J. Factores psicosociales y síndrome de burnout en personal de enfermería de una unidad de tercer nivel de atención a la salud. Cienc Trab. 2015; 17(52): 32-36.

14. Ministerio de la Protección Social. Primera Encuesta Nacional de Condiciones de Salud y Trabajo en el Sistema General de Riesgos Profesionales. Informe Final [internet]. Bogotá D.C: mps; 2007 [acceso el 27 de febrero de 2017]. Disponible en: http://www.ila. org.pe/publicaciones/docs/encuesta_nacional_colombia.pdf.

15. Ansoleaga M, Castillo A. Riesgo psicosocial laboral y patología mental en trabajadores de hospital. Revista Facultad Nacional de Salud Pública. 2011; 29(4): 372-379.

16. Sarsosa K, Charria V, Arenas F. Caracterización de los riesgos psicosociales intralaborales en jefes asistenciales de cinco clínicas nivel III de Santiago de Cali (Colombia). Rev. Gerenc. Polít. Salud. 2014; 13(27): 348-361.

17. Castillo I, Santana M, Valeta A, Alvis L, Romero E. Factores de riesgo psicosociales del trabajo en médicos de una Empresa Social del Estado en Cartagena de Indias. Revista Facultad Nacional de Salud Pública. 2011; 29(4): 363-371.

18. Castillo I, Torres N, Ahumada A, Cárdenas K, Licona S. Estrés laboral en enfermería y factores asociados. Cartagena (Colombia). Salud Uninorte. 2014; 30(1): 34-43.

19. Hilleshein E, Lautert L. Salud en el trabajo, la evaluación de la capacidad de trabajo, ambiente de trabajo y de enfermería. Rev. Latino-Am. Enfermagem. 2012; 20(3): 520-527.

20. Acevedo G, Sánchez J, Farías M, Fernández A. Riesgos Psicosociales en el Equipo de Salud de Hospitales Públicos de la Provincia de Córdoba, Santiago. Cienc Trab. 2013; 15(48): 140-147.

21. Ortiz F, Jaramillo V. Factores de riesgo psicosocial y compromiso (engagement) con el trabajo en una organización del sector salud de la ciudad de Cali, Colombia. Acta Colombiana de Psicología. 2013; 16(1): 43-56.

22. Ministerio de Salud (MINSAL). Primera Encuesta Nacional de empleo, trabajo, salud y calidad de vida de los trabajadores y trabajadoras de Chile. Informe interinstitucional. Dirección del Trabajo (DT), Instituto de Seguridad Laboral (ISL) ENETS 2009-2010.

23. González Y L. Evaluación de la percepción del riesgo ocupacional en trabajadores de una empresa del sector de la construcción en Bogotá DC. NOVA. 2015; 13 (23): 93 - 107.
24. Millan, J. and L. Yunda. An Open-Access Web-based medical image atlas for collaborative Medical image sharing, processing, Web Semantic searching and analysis with uses in medical training, research and second opinion of cases. 2014; Nova 12(22): 143-150.

25. Flórez, R. A. N. Avances y perspectivas en Síndrome de Asperger. 2014; Nova, 12(21). 